\title{
Erderkundung aus dem Weltraum - Das schweizerische ERTS- und EREP-Satellitenprojekt
}

\section{Einleitung}

Seit dem 23. Juni 1973 kreist der erste unbemannte Erderkundungssatellit ERTS-11) um unseren Planeten. Vom 26. Mai 1973 bis 8. Februar 1974 fanden die drei bemannten Missionen des SKYLAB statt mit Erderkundung (EREP-Programm) als einer der Hauptforschungsaufgaben. Am 22. Januar 1975 wurde der zweite unbemannte Erderkundungssatellit LANDSAT-2 erfolgreich auf seine Umlaufbahn gebracht. Die ersten beiden Satellitenprojekte ergaben eine Fülle von Datenmaterial und Forschungsergebnissen und erschlossen damit neue Anwendungsbereiche. Die Dateninterpretation ist aber noch längst nicht abgeschlossen, und schon verfügen wir über die ersten Aufnahmen des neuesten Satelliten. Es erscheint darum angebracht, heute kurz auf die allgemeine Bedeutung und die Möglichkeiten derartiger Satellitenprojekte für die Geographie einzugehen und in knapper Form über den Beitrag der Schweiz im Rahmen dieser weltweiten Forschungsaktivitäten zu berichten.

Wir sind in der bevorzugten Lage, daß die NASA für alle drei Experimente unsere schweizerischen Forschungsprojekte akzeptierte ${ }^{2)}$ und folglich eine regelmäßige Aufnahme der Schweiz gewährleistete. Damit verbunden ist eine kostenlose Abgabe des gesamten Bild- und Datenmaterials, das von unserem Land aufgenommen wird. Es ist zentral am Geographischen Institut der Universität Zürich archiviert.

An dieser Stelle kann nicht auf die vielschichtigen technischen Aspekte und Probleme der Satellitensysteme eingetreten werden. Wir verweisen auf die entsprechende Literatur (A/1-4). Nur die allerwichtigsten technischen Angaben sollen kurz zusammengefaßt werden:

ERTS-1): (Earth Resources Technology Satellite) Beinahe polare, sonnensynchrone Umlaufbahn, $80,9^{\circ}$ Inklination (Neigungswinkel zwischen Umlaufbahn und Äquator), Flughöhe $900-950 \mathrm{~km}$, ca. 14 Orbits pro Tag, Wiederholung derselben Umlaufbahn nach 18 Tagen, 4-Kanal-Multispektralscanner, erfaßt Bildstreifen von 185 km Breite, Bodenauflösung ca. 60-80 m, Schwarzweiß- und Farbaufnahmen, Digitaldaten auf Magnetband; ab Frühjahr 1974 nur noch «real-time»-Aufnahmen (über Aufnahmestationen in USA, Kanada und Brasilien und neuerdings auch Italien) möglich.
SKYLAB-EREP: Flugbahn von $435 \mathrm{~km}$ Höhe, $50^{\circ}$ Inklination (d. h. nur Gebiete bis $50^{\circ} \mathrm{N}$ resp. $\mathrm{S}$ erfaßt), ein Orbit in 95 Min., Repetition der gleichen Flugbahn alle fünf Tage, Originaldaten auf Erde zurückgebracht, Multibandkameras (ca. 30-70 m Bodenauflösung), Earth Terrain Camera (ca. $10 \mathrm{~m}$ Bodenauflösung), Infrarot-Spektrometer, 13-Kanal-Multispektralscanner, Altimeter, passive Mikrowellen-Radiometer und aktive Mikrowellen-Scatterometer.

LANDSAT-2: Gleiche Flugdaten und Aufnahmesysteme wie ERTS-1; gegenüber ERTS-1 um neun Tage versetzt, Äquatorüberquerung 9.32 h, 5 Min. später als ERTS-1. Seit 6. Februar 1975 routinemäßige Datenübermittlung.

\section{Vorteile, Möglichkeiten und Grenzen der Erkundungssatelliten}

Der Hauptvorteil besteht in der großräumigen wiederholten Erfassung der globalen Landfläche innert kurzen Zeitabständen (9-18 Tage), was eine ständig aktuell gehaltene Zustandserhebung bedeutet. Dadurch eröffnen sich ganz neue Forschungsbereiche, indem es erstmals möglich wird, rasch veränderliche Landschaftselemente und dynamische Landschaftsprozesse in ihrem räumlich-zeitlichen Ablauf über große Gebiete hinweg genau zu erfassen. Wichtig und dringlich gewordene Aufgaben wie die Inventarisierung aller Naturgüter, insbesondere der erneuerbaren Rohstoffe, als Grundlage für eine sinnvolle Planung und Nutzung, die Probleme der Umweltüberwachung und Umweltbelastung usw. lassen sich auf diesem Wege endlich in einem größeren, überregionalen Zusammenhang mit der nötigen Genauigkeit und innerhalb nützlicher Frist anpacken (A/5-7).

Eine Hauptaufgabe, die es anhand der Daten dieser experimentellen Satelliten zu lösen gilt, besteht in der Entwicklung von Methoden, um die skizzierten Aufgaben einmal routinemäßig mit operationellen Systemen durchführen zu können, die es ermöglichen, die notwendigen qualitativen und quantitativen Informationen zeitgerecht zu erhalten. Es müssen also Systeme

Prof. Harold Haefner, Geographisches Institut der Universität Zürich, Blümlisalpstraße 10, 8006 Zürich.

Prof. Bruno Messerli, Geographisches Institut der Universität Bern, Hallerstraße 12, 3012 Bern 
entwickelt werden, die einfach und ökonomisch aber auch schnell, präzis und allgemein einsetzbar sind, d.h. die die kontinuierlich eintreffende Datenflut im gleichen Tempo zu verarbeiten im Stande sind und praktisch «real-time»-Informationen zu liefern vermögen. Dies ist nur mit automatisierten Datenverarbeitungssystemen realisierbar.

Der Hauptnachteil für das ganze System bedeutet die Bewölkung, die vorab in unseren Breiten oft eine photographische Aufnahme der Erdoberfläche verunmöglicht. Eine Lösung läßt sich nur durch den Einsatz von Aufnahmesystemen erreichen, die Wolken zu durchdringen vermögen (aktive oder passive Mikrowellen).

Die bisher erzielten Ergebnisse und besonders auch das weitgespannte Spektrum der bearbeiteten Themen sind beachtlich. Wir verweisen hier auf die Berichte der europäischen und amerikanischen Fachtagungen (A/8-10).

\section{Aufnahmen der Schweiz}

Dank der offiziellen Forschungsprojekte wurde die Schweiz samt Umgebung 1972/73 regelmäßig vom ERTS-1 aufgenommen. Insgesamt wurden 40 Szenen (à je vier Multibandaufnahmen) von jeweils $185 \times 185 \mathrm{~km}$ $\left(34000 \mathrm{~km}^{2}\right)$ gemacht; davon sind ca. $2 / 3$ für erdkundliche, der Rest für meteorologische Zwecke brauchbar. Für eine Gesamtbedeckung der Schweiz sind je zwei Szenen an drei aufeinanderfolgenden Tagen notwendig. Leider konnte unser Land nie «en bloc» erfaßt werden, da Wetter und Aufnahmemodus (Prioritäten, limitierte Speicherkapazität) dies verunmöglichten. Die resultierende unsystematische Bedeckung erschwerte natürlich eine thematische Bearbeitung und ließ den Wunsch nach einer europäischen Empfangsstation für «real-time»-Aufnahmen aufkommen ${ }^{3)}$.

Das SKYLAB-Programm wurde von Beginn weg durch technische Schwierigkeiten beeinflußt, was zu einer Einschränkung der Forschungsprogramme führte, wovon besonders das EREP betroffen wurde4). Deshalb wurden die Alpen nur zweimal während der 2. bemannten Mission, am 11. September 1973 zwischen 15 h 26-27 und am 19. September 1973 mit dem gesamten Aufnahmeinstrumentarium erfaßt. Die Flugachse verlief vom Zentralmassiv über den Mt. Blanc, dem zentralen Wallis und Tessin ins Engadin. Das
Wetter war am ersten Überflug insbesondere in der Nordschweiz und in Graubünden sehr ungünstig, am zweiten Datum herrschte sogar 95\% Wolkenbedekkung, weshalb letztere Aufnahmen für geographische Untersuchungen unbrauchbar sind. Alle Aufnahmesysteme an Bord funktionierten einwandfrei.

\section{Die schweizerischen Forschungsprojekte}

Ausgehend von den in Kap. 2 allgemein formulierten Möglichkeiten der Satellitenbild-Interpretation wurde ein für die Schweiz und den alpinen Raum entsprechendes Forschungsprojekt erarbeitet, das von der aufnahmetechnischen Seite her den Einsatz verschiedener Spektralbereiche, von der zeitltchen her eine relativ langfristige Untersuchungsdauer, von der methodischen Fragestellung her vielseitige Ansätze und vom thematischen Standpunkt aus ein Eingehen auf die spezifischen Verhältnisse unseres Landes gestatten sollte. Das eingereichte Projekt mit dem Titel «Snow mapping and vegetation growth in the high mountains (Swiss Alps)», sollte vor allem die jahreszeitlichen Veränderungen im Hochgebirge vergleichend erfassen. Bedingt durch die erwähnten aufnahmetechnischen Schwierigkeiten wurden dann vor allem methodische Probleme zum zentralen Untersuchungsobjekt erhoben. Die einzige brauchbare EREP-Bedeckung verunmöglichte das Studium zeitlicher Veränderungen. So konnte primär der Zustand an einem bestimmten Datum erfaßt werden.

Trotz dieser aufnahmetechnischen Mängel, wie sie bei einem ersten Experiment stets auftreten können, darf das ganze ERTS-Projekt als ein Erfolg gewertet werden, der alle Erwartungen übertraf. Erfreulich war für uns ganz besonders, daß sich in der Schweiz bei der Auswertung der Satellitendaten eine echte interdisziplinäre Zusammenarbeit realisieren ließ, indem neben den beiden Geographischen Instituten auch das Eidg. Institut für Schnee- und Lawinenforschung, das Photographische Institut und das Kartographische Institut der ETHZ, die MZA und weitere Forscher aktiv daran beteiligt sind5). Gegenüber der NASA ist das ERTS-1Forschungsprojekt abgeschlossen (A/11), aber die Untersuchungen gehen natürlich weiter und lassen noch zahlreiche Bearbeitungsmöglichkeiten offen. Das Literaturverzeichnis (B) mit einer Zusammenstellung aller bisher in der Schweiz veröffentlichten Beiträge 
vermag einen Überblick über die verschiedenartig bearbeiteten Aspekte zu geben. Die nachfolgenden drei Beiträge sollen verschiedene Anwendungsmöglichkeiten der Interpretation von Satellitendaten aufzeigen. Dabei unterscheiden sich jeweils sowohl Problemstellung als auch Aufnahmematerial und Interpretationsmethode.

Mit dem LANDSAT-2-Programm möchten wir die begonnenen Untersuchungen fortsetzen und sowohl thematisch als auch regional sowie methodisch ausweiten. Mit Unterstützung eines vergrößerten Teams soll untersucht und geprüft werden, welche für die Raumplanung relevanten Informationen mittels Satellitendaten qualitativ und quantitativ erhoben werden können. Das Projekt trägt den Titel «Natural resources and land evaluation in Switzerland» und wurde im Frühjahr bereits in Angriff genommen.

\section{Anmerkungen}

1) Im Januar 1975 wurde das ERTS-Programm in "LANDSAT-Programm» umbenannt; die beiden Satelliten ERTS-1 und ERTS-B heißen jetzt entsprechend LANDSAT-1 und LANDSAT-2.

2) Gesamthaft wurden für LANDSAT-2 57 amerikanische und 55 nichtamerikanische Forschungsprojekte angenommen, darunter ca. 8 von Europa.

3) Es ist zu hoffen, daß dieser Wunsch mit der Empfangsstation Fucino bei Rom, die im März 1975 erstmals Daten empfangen konnte, in Erfüllung geht.

4) Durch Drehung des Himmelslabors von einer weltraum- in eine erdorientierte Position entstanden zusätzliche Energieprobleme.

5) Wir danken allen, die sich bei der Realisierung dieses Projektes tatkräftig eingesetzt haben, und ebenso dem Schweiz. Nationalfonds für die Finanzierung der Hauptuntersuchungen über die Methodik der Verarbeitung von multivariaten Digitaldaten.

\section{Literatur}

A) Allgemeine Literatur zum ERTS- und SKYLAB-Programm

1) ERTS DATA USERS HANDBOOK. NASA/GSFC Doc. No. 71SD4249, Washington D. C., 1972.

2) EREP USERS HANDBOOK. NASA, Manned Spacecraft Center, Houston, Texas, 1971.

3) SKYLAB Earth Resources Data Catalog. NASA, L. B. Johnson Space Center, Houston, Texas, 1974.

4) BANNERT, D. und MUEHLEFELD, R.: ERTS und SKYLAB - zwei erdumkreisende Erdforschungssysteme. Bildmessung und Luftbildwesen, No. 41/4, 1973.

5) BODECHTEL, J.: Möglichkeiten und zukünftige Entwicklung der Fernerkundung durch Satelliten. Kartographische Nachrichten, No. 24/3, 1974.

6) FISCHER, W. A.: Aerospace Methods for Revealing and Evaluating Earth's Resources. Paper UNo Seminar on Photogrammetric Techniques, Zürich, 1971.

7) HOWARD, J. A.: Satellite Sensing of Land Resources. Paper, FAO, AGS: Miss. 73/25, Rom, 1973.

8) ESRO: European Earth Resources Satellite Experiments. Proc. 1st Symp. on Europ. Earth Resources Satellite Experiments, Frascati, ESRO/Paris, 1974.

9) NASA/GSFC: Symposium on Significant Results Obtained from Earth Resources Technology Satellite-1. 2 Bde., Goddard Space Flight Center, Greenbelt, Maryland, 1973.

10) NASA/GSFC: Third Earth Resources Technology Satellite-1 Symposium. 2 Bde, Goddard Space Flight Center, Washington D. C., 1973.

11) HAEFNER, H.: ERTS-1 Final Report. Snow Survey and Vegetation Growth in High Mountains (Swiss Alps) and Additional ERTS-Investigations in Switzerland. Schlußbericht an NASA, Geogr. Institut Universität Zürich, 1975.

\section{B) Literatur zum schweizerischen ERTS- und EREP-Projekt}

GFELLER, R.: Untersuchungen zur automatisierten Schneeflächenbestimmung mit Multispektral-Aufnahmen des Erderkundungssatelliten ERTS-1, Diss. Univ. Zürich, 1975.

GFELLER, R. und SEIDEL, K.: Determination de la Couverture Neigeuse d'une Chaîne de Montagnes à l'Aide des Images reçues de Satellites. Coll. Int. LES SATELLITES METEOROLOGIQUES, Paris, 1973. 
HAEFNER, H.: Eyes above the Earth. ELEMENTS, No 9, Zürich, 1974.

HAEFNER, H., GFELLER, R. und SEIDEL, K.: Mapping of Snowcover in the Swiss Alps from ERTS-1 Imagery. COSPAR-Proceedings, 1973.

HAEFNER, H. und ITTEN, K.: National Report of Switzerland on Earth Resources Observation from Satellite Imagery. CosPAR-Proceedings, 1973.

HAEFNER, H. und SEIDEL, K.: Methodological Aspects and Regional Examples of Mapping Changes of Snowcover from ERTS-1 and EREP Imagery in the Swiss Alps. Proc. 1st Symp. on Europ. Earth Resources Satellite Experiments, Frascati, ESRO/Paris, 1974.

HERRMANN, Ch.: Entwicklungsmöglichkeiten topographischer Übersichtskarten (am Beispiel des Maßstabes 1:500000). Kartographische Nachrichten No. 23/4, 1973.

MEYER, J. H., KLEIN, P., HAEFNER, H., ITTEN, K. Und GFELLER, R.: Satellitenporträts der Schweiz. Tages-AnzeigerMagazin No. 37, 15. September, Zürich, 1973.

PIAGET, A.: First Preliminary Report on Meteorological Interpretation of Clouds or Cloud Systems Appearing on Pictures of the Alpine Region Received from the ERTS-1. Arbeitsberichte der Schweiz. Meteorologischen Zentralanstalt, Zürich, 1973.
PIAGET, A.: Interprétation Météorologique des Images à haute Résolution. Proc. 1st Symp. on Europ. Earth Resources Satellite Experiments, Frascati, ESRo/Paris, 1974.

SEIDEL, K., GFELLER, R. und BINZEGGER, R.: Snow and Vegetation Classification by Means of Digital LANDSATMSs-Data. Paper 4th Annual Remote Sensing of Earth Resources Conf., Tullahoma, Tennessee, 1975.

WINIGER, M.: Die raum-zeitliche Dynamik der Nebeldecke aus Boden- und Satellitenbildbeobachtungen. «Informationen und Beiträge zur Klimaforschung», No. 12, Geogr. Institut Universität Bern, 1974.

wINIGER, M.: Erderkundung aus der Luft und aus dem Weltraum. Die Welt von oben. Der Bund, No. 254, 30. Oktober 1973

WINIGER, M.: Klima- und Erdbeobachtung aus dem Weltraum. in: Messerli etal.: Beiträge zum Klima des Raumes Bern. Geographische Gesellschaft Bern, Bd. 50/1970-72.

im Druck:

BINZEGGER, R.: ERTS-Multispektraldaten als Informationsquelle für thematische Kartierungen. (Landnutzung im Raum Mailand.) Diss. Universität Zürich, 1975. 